\title{
An Experimental Study of Structural Identification of Bridges Using the Kinetic Energy Optimization Technique and the Direct Matrix Updating Method
}

\author{
Gwanghee Heo and Joonryong Jeon \\ Department of Civil and Environment Engineering, Konyang University, 121 Daehak-ro, Nonsan, Chungnam 320-711, Republic of Korea \\ Correspondence should be addressed to Joonryong Jeon; jrjeon@konyang.ac.kr
}

Received 25 September 2015; Accepted 27 December 2015

Academic Editor: Guillermo Rus

Copyright (C) 2016 G. Heo and J. Jeon. This is an open access article distributed under the Creative Commons Attribution License, which permits unrestricted use, distribution, and reproduction in any medium, provided the original work is properly cited.

\begin{abstract}
This paper aims to develop an SI (structural identification) technique using the KEOT and the DMUM to decide on optimal location of sensors and to update FE model, respectively, which ultimately contributes to a composition of more effective SHM. Owing to the characteristic structural flexing behavior of cable bridges (e.g., cable-stayed bridges and suspension bridges), which makes them vulnerable to any vibration, systematic and continuous structural health monitoring (SHM) is pivotal for them. Since it is necessary to select optimal measurement locations with the fewest possible measurements and also to accurately assess the structural state of a bridge for the development of an effective SHM, an SI technique is as much important to accurately determine the modal parameters of the current structure based on the data optimally obtained. In this study, the kinetic energy optimization technique (KEOT) was utilized to determine the optimal measurement locations, while the direct matrix updating method (DMUM) was utilized for FE model updating. As a result of experiment, the required number of measurement locations derived from KEOT based on the target mode was reduced by approximately $80 \%$ compared to the initial number of measurement locations. Moreover, compared to the eigenvalue of the modal experiment, an improved FE model with a margin of error of less than $1 \%$ was derived from DMUM. Thus, the SI technique for cable-stayed bridges proposed in this study, which utilizes both KEOT and DMUM, is proven effective in minimizing the number of sensors while accurately determining the structural dynamic characteristics.
\end{abstract}

\section{Introduction}

During their service period, structures are exposed not only to gradual aging but also to unspecified harmful environmental effects, such as earthquakes, strong winds, impacts, and structural instability due to external forces that can give rise to various structural defects. These defects can in turn lead to reduced life expectancy and unexpected serious structural damage. Thus, from a long-term perspective, continuous structural health monitoring (SHM), systematic evaluation, and maintenance efforts are pivotal, especially for structures that are vulnerable to vibration occurring during service, such as cable-stayed bridges. For these efforts, a technique for accurate structural identification (SI) is of utmost importance.

SI is defined as the process of defining a mathematical model of a given structure using measured physical information acquired from the actual target structure. Since Lie and Yao (1978) [1] introduced the concept of SI into structural engineering, many experiments have been conducted to identify a mathematical model that can represent the behavioral characteristics (equations of motion) of a target structure [2-5]. Despite such efforts, the SI technique has been applied in everyday practice in only a few cases and with limited effectiveness. It has not been adapted to convenient everyday application in general structural design and maintenance [6-8]. Appropriate utilization of the SI technique not only allows for thorough SHM of the target structure but also reduces the amount of effort required for evaluation and maintenance of the structure and can contribute to ensuring structural safety.

As explained above, to conduct SI, physical structural information must be obtained from the target structure through experimentation. In such experimentation, if 
the number of sensor locations can be selectively reduced such that all measurement locations are optimized to the target mode, both efficiency and economic viability can be improved from an administrative viewpoint. Soundness monitoring, which requires long-term, real-time measurements, involves figure-intensive data processing and requires repetitive operations. Thus, maximizing the quality of the monitored information while minimizing the number of sensors is of particular importance [9-16]. To achieve this, Kammer [13] used estimated error covariance matrix (EECM) to develop a new approach called the effective independence method (EIM). This method incorporates the concept of spatial independence and is typically applied to truss structures. EIM guarantees linear independence, and its calculations are very effective with regard to sensor location selection.

On the other hand, Gwanghee [12] proposed the kinetic energy optimization technique (KEOT) based on principles of structural kinetic energy under the premise that optimizing the composition and location of sensors by maximizing the kinetic energy (KE) of a structure would be the most effective way of determining damage in structures that feature low and close interval frequencies and high attenuation [911]. This method acquires optimal modal information by maximizing the kinetic energy measured from the structural system, thus ensuring the independence of the measuring mode used in KEOT. These types of advantages became handy when applied to large structures, thus proving the usefulness of KEOT [12].

Moreover, for SI, the mathematical model of the target structure must be defined using physical information measured from the target structure [17, 18]. In this process, an FE model that represents the structural state of the target structure is developed based on the measured data of the target structure. In addition, if the numerical modal parameters can be derived, they can be used to define the state of the baseline structure. At this point, it is typical for a certain margin of error to come into play with respect to the modeling. This is because the method of FE model interpretation can vary depending on factors such as lifespan, economics, expected value of the structural model, and the fact that it is constructed based on initial architectural drawings. FE models developed to particularly resolve dynamic structural problems must effectively predict changes in modal parameters caused by alterations in design parameters. In this regard, FE model improvement not only provides dynamic characteristics that conform to the target structure but also can be utilized in the future as an objective and reasonable standard based on which aging and localized damage to a structure can be measured $[19,20]$. In accordance with these needs, a range of FE model improvement methods [21] have been introduced. Namely, Friswell and Mottershead [22] have systematically organized classical theories such as the direct matrix updating method (DMUM) $[23,24]$ and the error matrix updating method (EMUM) $[25,26]$ and mathematically verified them to prove their validity. Thus, in this study, experiments were conducted to evaluate SI techniques aimed at accurately determining the modal parameters of a target structure based on optimal measurement locations (i.e., minimum number of sensors), which are the required parameters for efficient and practical SHM. To this end, the optimal sensor locations were selected based on a model of a cable-stayed bridge and then the responses from the selected measurement locations were used to improve the FE model. Taking into account the structural characteristic of cablestayed bridges, which feature relatively natural frequency of narrow interval and short cycle, KEOT was applied to select the optimal sensor locations. Moreover, to improve the FE model, DMUM was utilized because it requires fewer calculations, has high accuracy, and is practical for everyday use.

In conclusion, for structures such as cable-stayed bridges where the characteristic flexing behavior creates a natural frequency of narrow interval and short cycle, situations where multiple sensors are required because of structural complexity, and situations where there exist uncertainties in the FE model used for interpreting the data, KEOT and DMUM can facilitate rational and effective SI by enabling identification of the optimal sensor locations and improvement of the FE model. Ultimately, this study proves through experimentation that a combination of KEOT and DMUM is the ideal hybrid method to provide both practical and effective SHM.

\section{Optimal Sensor Location and Finite Element (FE) Model Update}

2.1. Kinetic Energy Optimization Technique (KEOT). In this study, the KEOT method was applied and evaluated for its effectiveness in selecting optimal sensor locations. KEOT recommends selecting optimal measurement locations by utilizing the strain-kinetic energy of the structure [9-12]. To do this, Kammer's EIM approach [13] involves optimizing and selecting a set of target modes in order to identify the structure based on FE analysis. An initial candidate set of transducer locations is also selected. These locations are then ranked based on their contribution to the linear independence of the corresponding FE model target mode partitions, and locations that do not contribute are removed from the candidate set. The described energy optimization technique algorithm is a modification of EIM, and it is designed to improve the modal information and maximize the measured kinetic energy of the structural system. The spatial independence of the identified mode shapes is satisfied by the sensing configuration obtained using the KEOT algorithm $[11,12]$. The kinetic energy in the system is

$$
\mathrm{KE}=\Phi^{T} M \Phi,
$$

where $\Phi$ is the measured mode shape vector. After decomposing the mass matrix $M$ into upper and lower triangular Cholesky factors, the kinetic energy matrix can be derived as

$$
\mathrm{KE}=\Psi^{T} \Psi,
$$

where $\Psi=U \Phi$ and $M=L U$. The matrices $L$ and $U$ denote the lower and upper triangular Cholesky factors. 
The projection of the mode shapes onto the reduced configuration is denoted by

$$
\begin{aligned}
& \bar{\Phi}=\text { projection }(\Phi), \\
& \bar{\Psi}=\operatorname{projection}(\Psi) .
\end{aligned}
$$

Similarly, the energy measured by the reduced set of transducers is obtained from the initial energy by removing the contribution of all transducers that have been eliminated:

$$
\overline{\mathrm{KE}}=\bar{\Psi}^{T} \bar{\Psi} .
$$

The objective of the transducer placement is to find a reduced configuration that maximizes the measurement of the kinetic energy of the structure. It is desirable to stop eliminating the transducers when doing so results in a rank deficiency of the energy matrix. Assuming that the mass matrix is nonsingular, the column rank $N$ of the quantity $\overline{\mathrm{KE}}$ is equal to the number of linearly independent projected vectors in matrix $\bar{\Phi}$. The problem is solved iteratively by the following procedure. First, the eigenvalues $\Lambda$ and eigenvectors $\psi$ of the energy matrix are extracted:

$$
\overline{\mathrm{KE}} \psi=\Lambda \psi .
$$

Computing the eigenpairs at each iteration of the energy optimizing technique (EOT) procedure does not significantly increase the computational cost because the matrix $\overline{\mathrm{KE}}$ is a square, symmetric, and positive-definite matrix of size $N$. Then, using an approach similar to Kammer's EIM [13], the fractional contributions of each remaining transducer are assembled into the KEOT vector:

$$
\mathrm{KEOT}=\sum_{i=1, \ldots, m}\left[\bar{\Psi} \psi \Lambda^{-1 / 2}\right]^{2} .
$$

The transducer location with the minimum contribution in the KEOT vector is then selected for removal. Subsequently, the contribution of the removed transducer to the kinetic energy matrix is deleted, and the new matrix is checked for rank deficiency. If the removal of the transducer produces a rank deficiency, it implies that the transducer location in question cannot be removed. If removing the transducer does not produce a rank deficiency, the transducer location is removed from the candidate set and the process is repeated until the required number of transducers is ascertained. Because it can be verified that the quantity between the brackets in (6) represents a linear combination of the measured mode shapes that is designed to produce orthogonal vectors,

$$
\left[\bar{\Psi} \psi \Lambda^{-1 / 2}\right]^{T}\left[\bar{\Psi} \psi \Lambda^{-1 / 2}\right]=I .
$$

Furthermore, each KEOT of the vector is a heuristic measure of the contribution of each transducer to the measured energy. The normalization factor $\Lambda^{-1 / 2}$ prevents the contributions of high frequency modes from dominating the low frequency modes. In theory, the number of remaining transducers is equal to the size of the target modal set. However, the apparent rank is often increased due to noise in the experimental data, and more than $M$ transducers are required to identify $N$ independent modes.
2.2. Direct Matrix Updating Method (DMUM). In general, when a modified structure is added to or removed from the initial structure, changes occur in the dynamic characteristics of the structure. Dynamic characteristics before and after a structural change can be derived as eigenvalue formulas shown below:

$$
\begin{array}{r}
([K]-\Lambda[M])\{\Phi\}=0 \\
(([K]+[\Delta K])-\bar{\Lambda}([M]+[\Delta M]))\{\bar{\Phi}\}=0
\end{array}
$$

where $[K]$ is the stiffness matrix, $[M]$ is the mass matrix, and $[\Delta K]$ and $[\Delta M]$ are changes in the stiffness and mass of the structure, respectively, due to structural changes. The elements $\Lambda$ and $\{\Phi\}$ are eigenvalue and eigenvector, respectively, and $\bar{\Lambda}$ and $\{\bar{\Phi}\}$ are changes in eigenvalue and eigenvector due to structural changes, respectively. Regarding the derivation methods available for calculating changes in stiffness $[\Delta K]$ and mass $[\Delta M]$ due to structural changes, this study selected and applied DMUM considering its effectiveness in FE model improvement as well as its convenience and practicality for everyday use. Here, using Lagrange multipliers, objective functions, composed to limit the range of change in the stiffness and mass matrices while simultaneously satisfying the eigenvalue, are shown below as (9). DMUM is advantageous from the perspective of usability because it can calculate the changes in stiffness and mass from just a single matrix operation $[23,24]$ :

$$
\begin{aligned}
\epsilon_{K} & =\left\|\left[K_{A}\right]^{-1 / 2}\left(\left[K_{U}\right]-\left[K_{A}\right]\right)\left[K_{A}\right]^{-1 / 2}\right\|, \\
\epsilon_{M} & =\left\|\left[M_{A}\right]^{-1 / 2}\left(\left[M_{U}\right]-\left[M_{A}\right]\right)\left[M_{A}\right]^{-1 / 2}\right\|,
\end{aligned}
$$

where $\left[K_{A}\right]$ and $\left[M_{A}\right]$ are the stiffness and mass matrices before structural change and $\left[K_{U}\right]$ and $\left[M_{U}\right]$ are the stiffness and mass matrices after structural change. The correlation between $\left[K_{A}\right]$ and $\left[K_{U}\right]$ can be expressed as shown in (10), and the correlation between $\left[M_{A}\right]$ and $\left[M_{U}\right]$ can be expressed as shown in (11):

$$
\begin{aligned}
& {\left[K_{U}\right]=\left[K_{A}\right]+[\Delta K],} \\
& {\left[M_{U}\right]=\left[M_{A}\right]+[\Delta M] .}
\end{aligned}
$$

The element $[\Delta K]$ in (10) can be defined as (14) using the interaction formulas of (12) and (13) [27]:

$$
\begin{aligned}
& {\left[K_{U}\right]-\left[K_{U}\right]^{T}=0,} \\
& {\left[\Phi_{X}\right]^{T}\left[K_{U}\right]\left[\Phi_{X}\right]-\left[\Lambda_{X}\right]=0,} \\
& {[\Delta K]=-\left[K_{A}\right]\left[\Phi_{X}\right]\left[\Phi_{X}\right]^{T}\left[M_{A}\right]} \\
& \quad-\left[M_{A}\right]\left[\Phi_{X}\right]\left[\Phi_{X}\right]^{T}\left[K_{A}\right] \\
& \quad+\left[M_{A}\right]\left[\Phi_{X}\right]\left[\Phi_{X}\right]^{T}\left[K_{A}\right]\left[K_{A}\right]\left[\Phi_{X}\right]\left[\Phi_{X}\right]^{T}\left[M_{A}\right] \\
& \quad+\left[M_{A}\right]\left[\Phi_{X}\right]\left[\Lambda_{X}\right]\left[\Phi_{X}\right]^{T}\left[M_{A}\right] .
\end{aligned}
$$


The element $[\Delta M]$ in (11) can be defined as (17) using the interaction formulas of (15) and (16) [28]:

$$
\begin{aligned}
& {\left[M_{U}\right]-\left[M_{U}\right]^{T}=0} \\
& {\left[\Phi_{X}\right]^{T}\left[M_{U}\right]\left[\Phi_{X}\right]-[I]=0} \\
& {[\Delta M]=-\left[M_{A}\right]\left[\Phi_{X}\right]\left(\left[\Phi_{X}\right]^{T}\left[M_{A}\right]\left[\Phi_{X}\right]\right)} \\
& \quad+\left[\Phi_{X}\right]^{T}\left[K_{A}\right] \\
& \quad+\left([I]-\left[\Phi_{X}\right]^{T}\left[M_{A}\right]\left[\Phi_{X}\right]\right)\left(\left[\Phi_{X}\right]^{T}\left[M_{A}\right]\left[\Phi_{X}\right]\right)
\end{aligned}
$$

In this study, the objective of FE model improvement is FE interpretation and modal experiment results, so the subscript $A$ in each equation is the result value based on analysis and subscript $X$ is the result value based on experimentation.

\section{FE Analysis and Modal Test of Model Structure}

3.1. Setup of the Model Structure (Cable-Stayed Bridge). The second moment of inertia of the cable-stayed bridge model used in this study was reduced as much as possible so that the bridge slab would be sensitive to vibrations caused by external forces. Moreover, to make FE model configuration more convenient, the cross-sectional shape and the materials used for the members were simplified and unified. Further, to reduce the possibility of a production error, cross section dimensions were equalized and initial changes were minimized. The model used in this study is a three-span continuous beam structure with a total length of $4220 \mathrm{~mm}$, a width of $170 \mathrm{~mm}$, and a pylon height of $1000 \mathrm{~mm}$. The cables are $0.8 \mathrm{~mm}$ steel wires, with ten wires connected to corresponding points on the crossbeam from each side of the pylon. The anchors located at each end of the model were composed of rollers, and the anchors of the pylons were composed of rollers and hinges. Finally, $1 \mathrm{~kg}$ of additional mass was added to the top of the bridge slab at each of the 39 points where the wires join the cross beam to maximize the flexibility of the bridge without creating any initial deflections. The model built using the components explained above is shown in Figure 1, and detailed design data is shown in Table 1.

During the assembly of the model, the cables were tensioned taking into consideration the effect of gravity on the superstructure across all sections. Thus, the selfweight of the superstructure was assumed to be a uniformly distributed load distributed at equal intervals across the cross beams and was utilized as the tensile force of the cables. To quantify the tensile force, a force gauge was utilized when tensioning the cables. After the completion of cable tensioning, a level was used to inspect the horizontality of the superstructure. In addition, the cables were tensioned progressively outward from the pylons and symmetrically left and right in accordance with the cable tensioning order of an actual cable-stayed bridge structure, as shown in Figure 2.

3.2. FE Analysis of Model Structure (Cable-Stayed Bridge). In order to analyze the structural dynamic properties of
TABLE 1: Design data of the cable-stayed bridge model.

\begin{tabular}{lc}
\hline Category & Data \\
\hline Total length & $4.22 \mathrm{~m}$ \\
Length of the center span & $2.22 \mathrm{~m}$ \\
Length of each side span & $1.0 \mathrm{~m}$ \\
Width of the superstructure & $0.17 \mathrm{~m}$ \\
Pylon height & $1.00 \mathrm{~m}$ \\
Pylon anchor type & Roller and hinge (Pin) \\
Bridge end anchor type & Roller \\
Diameter of cable & $0.8 \mathrm{~mm}$ \\
\hline
\end{tabular}

the produced model bridge, in this paper, FE modelingbased numerical analysis was conducted. For this purpose, IDEAS from UGS, a commercial structural analysis program, was used, and a three-dimensional detailed FE model was organized as in Figure 3 by applying the physical properties of Table 2. Here, $1 \mathrm{D}$ beam element was considered for the bridge deck and the tower of the structure, rigid element was considered for the bridge deck boundary conditions, and 1D rod element was considered for cable; as for the floor beam and lumped mass, $1 \mathrm{~kg}$ size of lumped mass was considered for each of the 39 nodes, which are the positions of the floor beams on the area of the central bridge deck excluding the pylon and both sides. Next, as for the boundary condition, clamp was considered for the substructure of the pylon, roller was considered for the both sides of superstructure and the right-side pylon connection, and Pin was considered for the left-side pylon connection. Lastly, as for the freedom degree condition of the structure, DOF was given in the direction of $y$-axis with the 39 nodes on the central bridge deck as the base to complete the three-dimensional detailed FE model. In this context, the FE analysis of the model bridge considered a total of two lowest flection modes for the purpose of vertical vibration control, and the natural frequency and mode shape of the model bridge that has been analyzed with Guyan reduction method applied are as in Figure 4.

In this paper, the DOFs of FE model were reduced into thirty-nine, with application of Guyan reduction method [29] by which a structural analysis of eigenvalues is made by reducing removed DOFs into unremoved ones and in turn composing a matrix of reduced strength and mass as in (18). Therefore, it has become possible to reduce the DOFs by selecting a desired point of measurement and to effectively perform structural FE analysis. Here, each $r$ refers to a retained set and $o$ to an omitted set. Equation (18) is applied for an analysis of eigenvalues, based on an optimal location of measurement determined in Section 4.1:

$$
\begin{aligned}
K_{r}= & K_{r r}-K_{r o} K_{o o}^{-1} K_{r o}^{T}, \\
M_{r}= & M_{r r}-M_{r o} K_{o o}^{-1} K_{r o}^{T}-K_{r o} K_{o o}^{-1} M_{r o}^{T} \\
& +K_{r o} K_{o o}^{-1} M_{o o} K_{o o}^{-1} K_{r o}^{T} .
\end{aligned}
$$

As shown in Figure 4, based on the total of 39 DOF, Guyan reduction method was applied to interpret the frequency of 
TABLE 2: Material properties of the cable-stayed bridge model.

\begin{tabular}{lcccc}
\hline $\begin{array}{l}\text { Material } \\
\text { property }\end{array}$ & $\begin{array}{c}\text { Modulus of } \\
\text { elasticity } \\
\left(\mathrm{kgf} / \mathrm{mm}^{2}\right)\end{array}$ & $\begin{array}{c}\text { Shear modulus of } \\
\text { elasticity } \\
\left(\mathrm{kgf} / \mathrm{mm}^{2}\right)\end{array}$ & $\begin{array}{c}\text { Poisson's } \\
\text { ratio }\end{array}$ & $\begin{array}{c}\text { Unit weight } \\
\left(\mathrm{kgf} / \mathrm{mm}^{3}\right)\end{array}$ \\
\hline Steel & $2.10 \times 10^{4}$ & $8.10 \times 10^{3}$ & 0.30 & $\begin{array}{c}\text { Yield } \\
\text { strength } \\
\left(\mathrm{kgf} / \mathrm{mm}^{2}\right)\end{array}$ \\
\hline
\end{tabular}

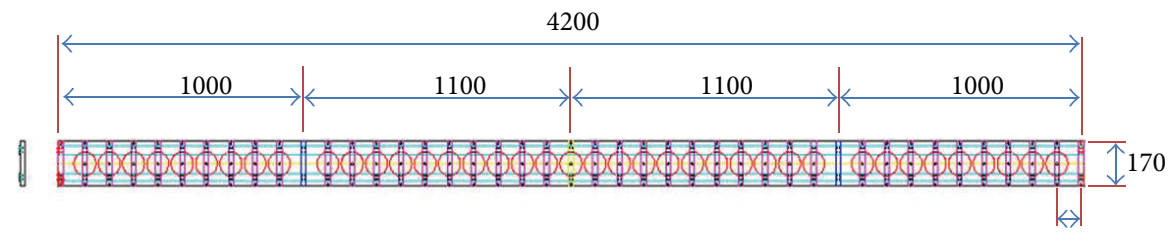

100
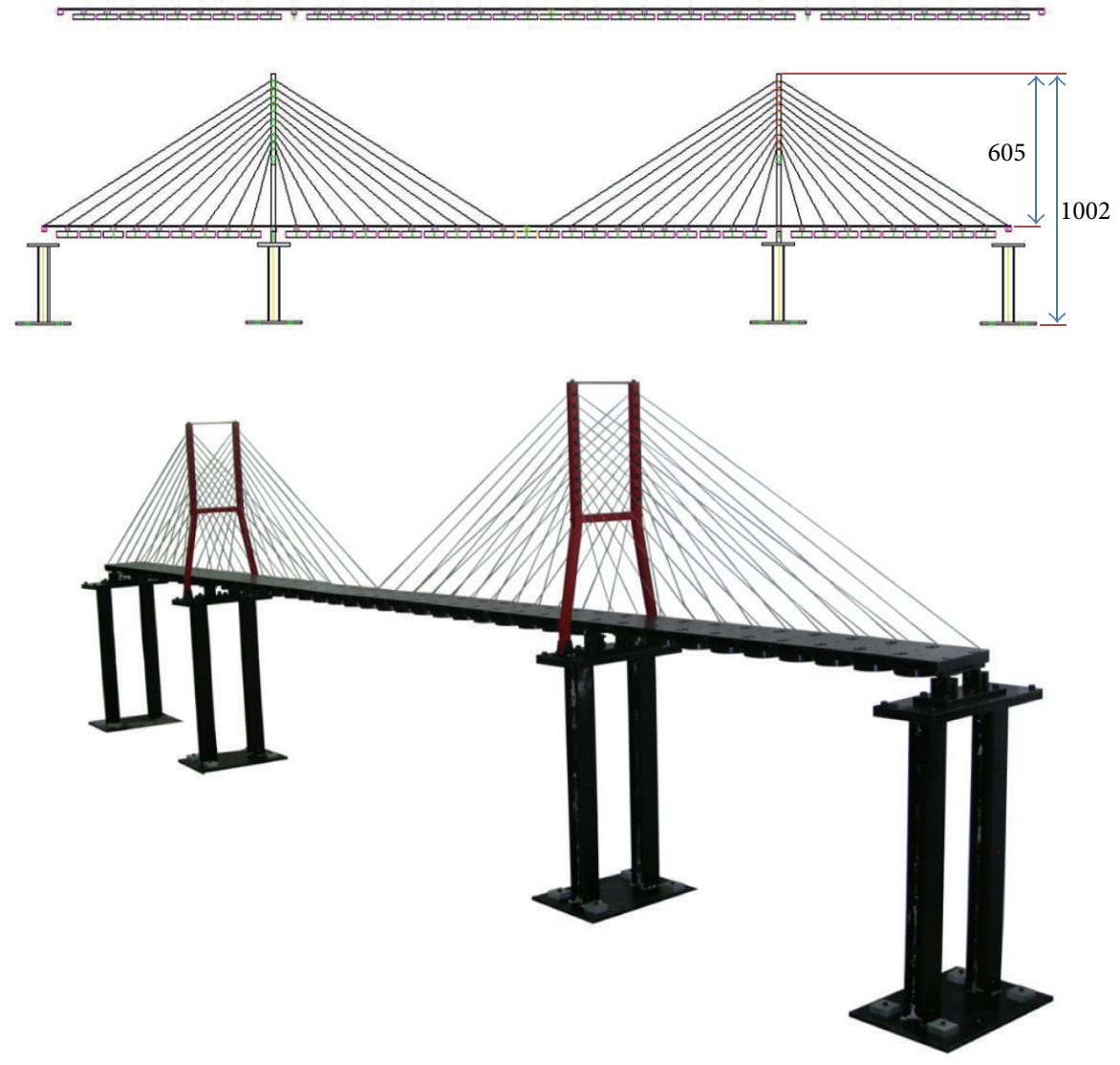

FIGURE 1: View of the cable-stayed bridge model.

vibration and the mode shape, with the results shown in Table 3.

3.3. Modal Test of the Model Structure (Cable-Stayed Bridge). To confirm the validity of the composed FE model and to analyze the fundamental kinetic characteristics of the model bridge, this study employed a modal test conducted using an impact hammer. An HP-VXI 1432 was used to measure the response signals from the structure, and data was obtained and analyzed by utilizing T-DAS by MTS. In order to obtain acceleration responses of the structure, Dytran model 3134D was used for a total of 39 points on the bridge deck that had been selected in equal intervals. To connect the accelerometer to the experimental bridge model and the measurement system, $10 \mathrm{ft}$ cables were used for each channel. The impact hammer used to create vibratory forces in the structure was a $5850 \mathrm{~A}$ model produced by Dytran, and the center of the slab was selected as the location of the impact.

The size of each frame of the measured frequency response function (FRF) was set to 2048, and the maximum frequency range was set to $35 \mathrm{~Hz}$. Data was collected by averaging the results of 30 tests. Finally, the acquired time and 


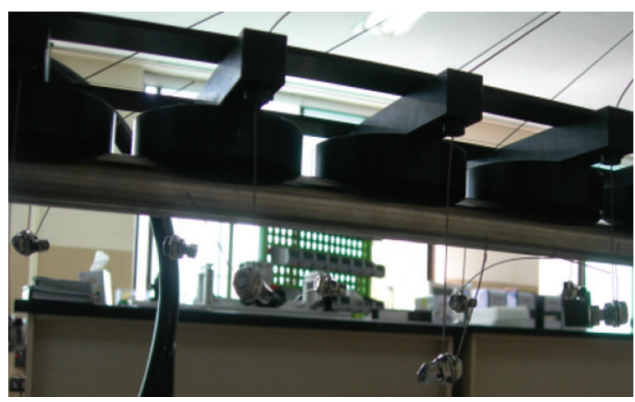

(a) Jig for cable tensioning

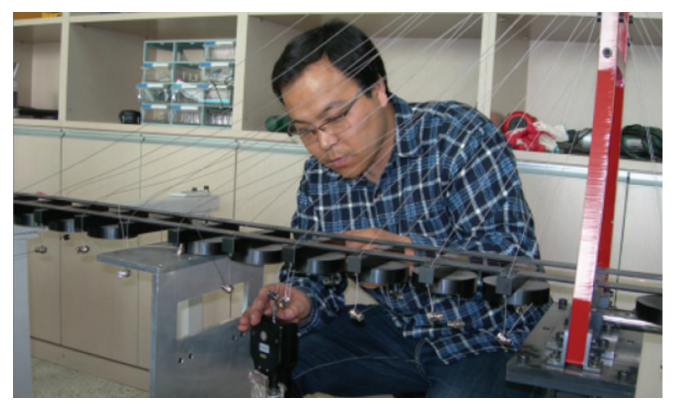

(b) Tensioning the cables

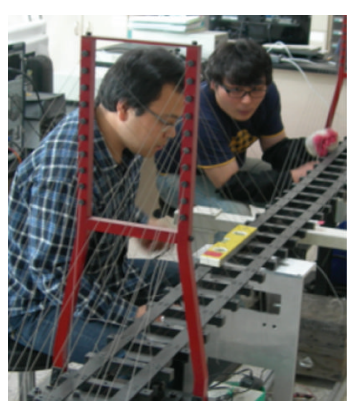

(c) Inspecting the horizontality

FIGURE 2: Steps for tensioning the cables of the cable-stayed bridge model.

TABLE 3: FE analysis results of the cable-stayed bridge model.

\begin{tabular}{lllll}
\hline & 1st bending & 2nd bending & 3rd bending \\
Analysis \\
results
\end{tabular}

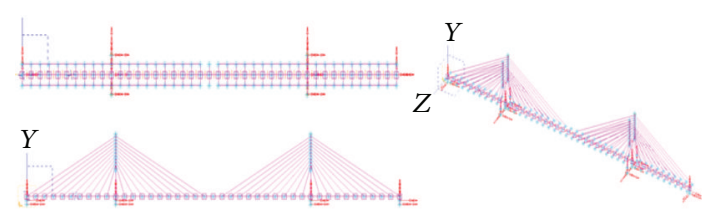

FIGURE 3: Initial FE modeling of the model bridge using I-DEAS.

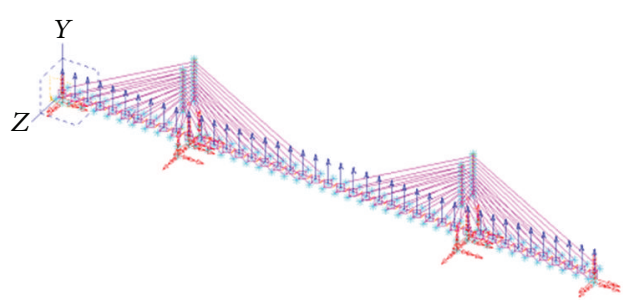

FIGURE 4: Initial FE modeling of the model bridge using I-DEAS (39 DOF).

frequency responses are as shown in Figure 5, and the results of the modal test analysis are as shown in Table 4.

To confirm the validity of using the FE model to understand the dynamic behavior characteristics of the model bridge and for use in deriving the modal parameters for future SHM and maintenance, this study compares and contrasts the frequency results of the FE model analysis and the modal experiment as shown in Table 5. As observed from the data in the table, reliable results were achieved, with an error ratio of around $1 \%$ between the $\mathrm{FE}$ analysis and the modal experiment. These results prove that the detailed FE model constructed in this study reliably mimicked the behavioral characteristics of the actual structural model, thereby confirming its validity.
The analysis and experimental results above were acquired using a total of 39 DOF and sensors. From the standpoint of long-term continuous SHM and from the practical standpoint of everyday evaluation and maintenance, using the whole structure to acquire signals as done in this study may be inefficient and impractical. Doing so would require a great number of sensors as well as a great deal of labor to install them. Moreover, interpreting and sorting through the resulting massive amount of data would be a laborious task. To resolve this problem, an alternative approach is required. This study therefore considers an approach whereby optimal sensor locations are selectively chosen in order to acquire the maximum information about the behavioral characteristics of the structure from a minimum number of sensors. By selecting the optimal locations, the sensors can thus be positioned at the most rational and efficient points to acquire the target information. Moreover, the sensor locations selected through this approach can later be utilized as real-time sensor locations for actual structures.

\section{Optimal Sensor Location and FE Model Updating of the Model Bridge}

4.1. Optimal Sensor Location Using the KEOT. The aim of this study is to conduct SI with a limited number of sensors by selecting the optimal sensor locations. To select the optimal sensor locations, we utilized KEOT, which maximizes the structure's strain-kinetic energy to enable consideration of the optimal measurement conditions. The KEOT method has proven to be effective for ascertaining the kinetic parameters of structures that feature low and short-interval frequencies, such as the cable-stayed bridge used in this study [9-12]. In this study, we set out to use KEOT to select the optimal sensor locations to represent the three lowest bending modes 


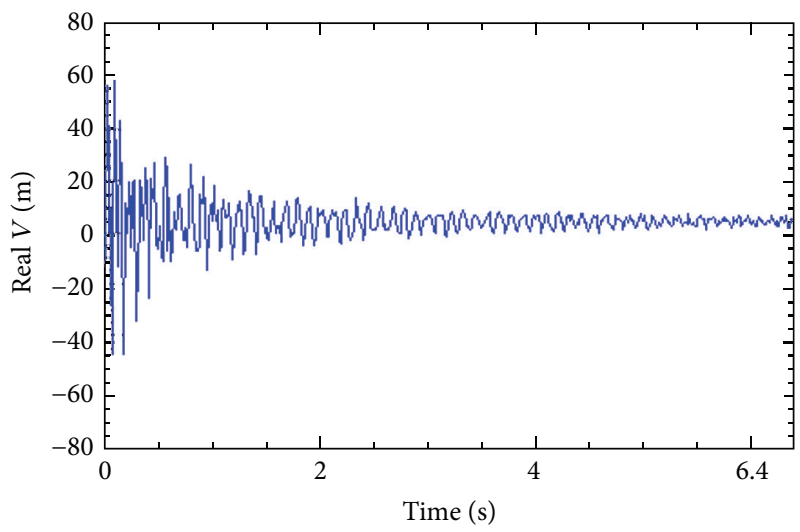

(a) Modal test response (time)

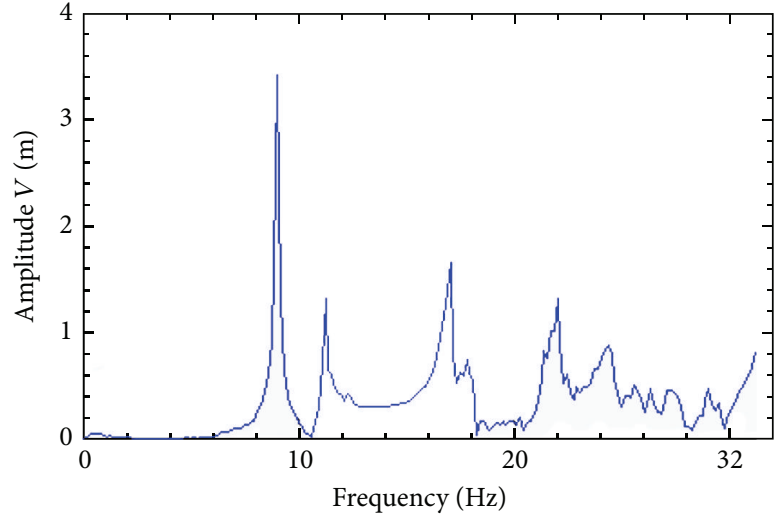

(b) Modal test response (spectra)

FIGURE 5: Modal test results.

TABLE 4: Results of the modal test of the cable-stayed bridge model.

Modal
test
results

TABLE 5: Comparison of analysis and experimental frequency.

\begin{tabular}{lccr}
\hline & & Analysis and experimental results & \\
& FE analysis results $(\mathrm{Hz})$ & Modal experiment results (Hz) & Error ratio (\%) \\
\hline 1st bending mode & 9.1719 & 11.2557 & 0.9120 \\
2nd bending mode & 11.2544 & 17.1442 & 0.0115 \\
3rd bending mode & 16.8643 & 1.0326 \\
\hline
\end{tabular}

TABLE 6: FE analysis results per DOF of cable-stayed bridge model using EOT.

\begin{tabular}{|c|c|c|c|c|c|c|c|}
\hline \multicolumn{2}{|c|}{ Target mode } & \multicolumn{2}{|c|}{ 1st bending } & \multicolumn{2}{|c|}{ 2nd bending } & \multicolumn{2}{|c|}{ 3rd bending } \\
\hline $\mathrm{DOF}$ & Reduction (\%) & Freq. $(\mathrm{Hz})$ & Error (\%) & Freq. $(\mathrm{Hz})$ & Error (\%) & Freq. $(\mathrm{Hz})$ & Error $(\%)$ \\
\hline 39 (full) & - & 9.1719 & - & 11.2544 & - & 16.8643 & - \\
\hline 30 & 23.07 & 9.1728 & 0.0009 & 11.2567 & 0.0204 & 16.8691 & 0.0284 \\
\hline 25 & 35.89 & 9.1757 & 0.0414 & 11.2651 & 0.0950 & 16.8856 & 0.1263 \\
\hline 20 & 48.71 & 9.1890 & 0.1864 & 11.3049 & 0.4487 & 16.9834 & 0.7062 \\
\hline 15 & 61.53 & 9.2219 & 0.5451 & 11.7537 & 4.4364 & 17.2843 & 2.4904 \\
\hline 10 & 74.35 & 9.3253 & 1.6724 & 11.7537 & 4.4364 & 18.1914 & 7.8692 \\
\hline 5 & 87.17 & 9.5531 & 4.1561 & 12.3951 & 10.1355 & 18.8781 & 11.9412 \\
\hline
\end{tabular}

among the 39 DOF nodes. The three lowest bending modes are important and interesting modes that can occur during vertical vibration and thus should be the target modes for SI. Also, the optimal sensor locations were selected with a low error rate based on these target modes. Figure 6 and Table 5 show the optimal sensor locations selected and the results of interpretation using the KEOT method compared to the rate of error in frequency evaluated per DOF node. Rank deficiency in the energy matrix did not occur until the 39 sensor locations had been reduced to 2 . This study therefore limited the final reduced DOF count to 5 and selected the optimal sensor locations based on analysis of changes in eigenvalue.

By observing the trends in optimal sensor locations selected through gradual removal of DOF nodes (as shown in Table 6), it can be observed that when the total of 39 locations had been reduced to 10 (at the $75 \%$ reduction point), all of the left- and right-side span sensor locations had been removed. Thus, it was concluded that the strain-kinetic energy due to the 1st, $2 \mathrm{nd}$, and 3rd bending mode behaviors was smaller in the side spans than in the center span. On the other hand, within the center span, the remaining sensor 


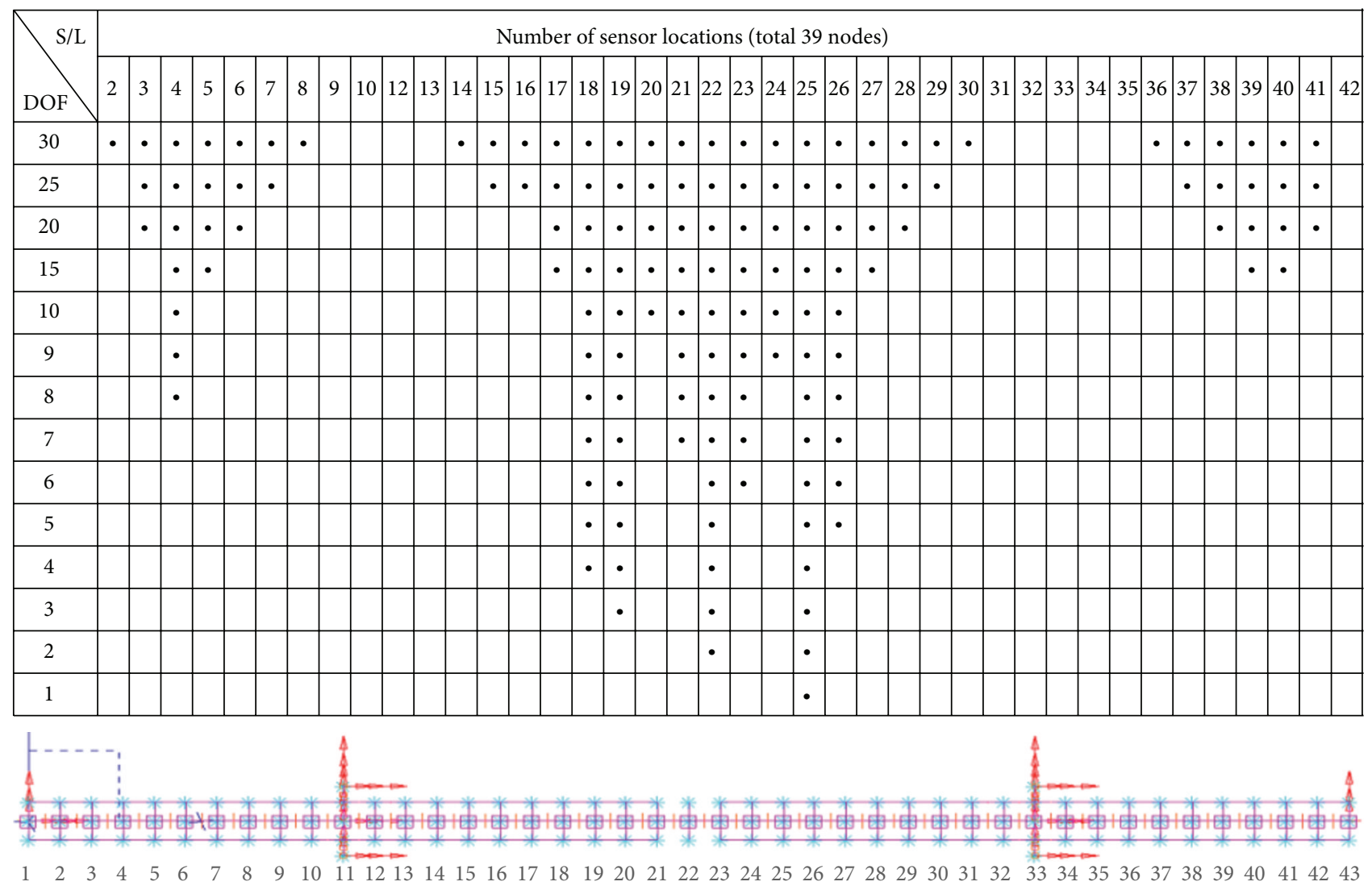

FIGURE 6: Study of optimal sensor locations selected using the EOT method.

locations grouped around nodes 19, 22, and 25 accounted for all the 1st, $2 \mathrm{nd}$, and $3 \mathrm{rd}$ bending modes. These three nodes remained until only three sensor locations were left (at the $92.5 \%$ reduction point). Thus, it was concluded that nodes 19,22 , and 25 were the essential sensor locations that can most optimally represent the 1st, 2 nd, and 3rd bending modes simultaneously. In conclusion, for comprehensive representation of the 1st, $2 \mathrm{nd}$, and $3 \mathrm{rd}$ resonance modes, which have the greatest impact on bending, it is essential that these three sensor locations are included.

On the other hand, when sensor locations or DOF nodes are removed using the KEOT method explained above, changes in eigenvalue can be impacted due to the reduction in DOF. This study evaluated changes in eigenvalue when DOF nodes are removed using KEOT. Table 6 shows the results of eigenvalue analysis when the $39 \mathrm{DOF}$ nodes are reduced to $30,25,20,15,10$, and finally 5 .

As shown in Table 6, changes in eigenvalue were clearly observed as the total of 39 DOF nodes was reduced to 25,25 , $20,15,10$, and 5 . Looking closely at the changes in frequency, it was also observed that, with the reduction in the number of DOF nodes, the frequency steadily increased. In the case of the 1st bending mode, the frequency error ratio significantly increased when DOF nodes were reduced to 10 (reduction rate of about $75 \%$ ) and the error ratio exceeded $4 \%$ when they were reduced to 5 (reduction rate of about $87 \%$ ). In the case of the 2 nd bending mode, the frequency error ratio increased significantly when the DOF nodes were reduced to 15 (reduction rate of about $60 \%$ ). It was close to $4.5 \%$ when the nodes were reduced to 10 (reduction rate of about $75 \%$ ) and exceeded $10 \%$ when the nodes were reduced to 5 (reduction rate of about $87 \%$ ). Moreover, in the case of the 3 rd bending mode, the frequency error ratio significantly increased when the DOF nodes were reduced to 15 (reduction rate of about $60 \%$ ). It was close to $8 \%$ when the nodes were reduced to 10 (reduction rate of about $75 \%$ ) and reached about $12 \%$ when the nodes were reduced to 5 (reduction rate of about $87 \%$ ).

These tendencies are due to the fact that stiffness is the most considered factor in the process of condensing the behavioral characteristics of an entire structure to a limited number of DOF nodes. In conclusion, to derive FE analysis results using Guyan reduction [29], it is important to select the range of allowable error ratio and the corresponding reduction of DOF nodes. Otherwise, excessive reduction in the number of DOF nodes will increase the frequency error ratio and lead to discrepancies in the initial FE analysis and modal test results, ultimately producing distorted frequency results.

Using the above results, a total of seven sensor locations were selected to include the three optimal sensor locations 19,22 , and 25 , to limit the frequency error ratio to less than $10 \%$ and to clearly observe the shape of the modes. Using the KEOT method, the optimal sensor locations selected were 18 , $19,21,22,23,25$, and 26. 
TABLE 7: Frequency and mode shape of 7 DOF using KEOT.

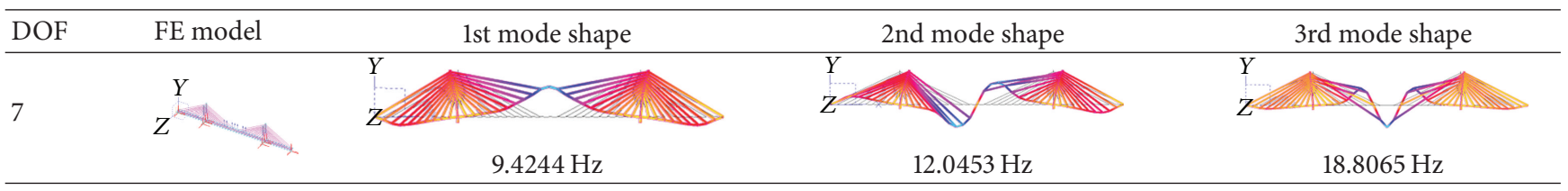

TABLE 8: Comparison of 7 DOF analysis results and full DOF cases.

\begin{tabular}{|c|c|c|c|c|c|c|}
\hline & \multicolumn{3}{|c|}{ Analysis (7 DOF) versus analysis (39 DOF) } & \multicolumn{3}{|c|}{ Analysis (7 DOF) versus experimental (39 DOF) } \\
\hline & $\begin{array}{l}\text { Analysis } \\
\text { (7 DOF) }\end{array}$ & $\begin{array}{l}\text { Analysis } \\
\text { (39 DOF) }\end{array}$ & Error $(\%)$ & $\begin{array}{l}\text { Analysis } \\
\text { (7 DOF) }\end{array}$ & $\begin{array}{c}\text { Experimental } \\
(39 \mathrm{DOF})\end{array}$ & Error $(\%)$ \\
\hline 1st mode & $9.4244 \mathrm{~Hz}$ & $9.1719 \mathrm{~Hz}$ & 2.7529 & $9.4244 \mathrm{~Hz}$ & $9.0890 \mathrm{~Hz}$ & 3.6900 \\
\hline 2nd mode & $12.0453 \mathrm{~Hz}$ & $11.2544 \mathrm{~Hz}$ & 7.9160 & $12.0453 \mathrm{~Hz}$ & $11.2557 \mathrm{~Hz}$ & 7.0151 \\
\hline 3rd mode & $18.8065 \mathrm{~Hz}$ & $16.8643 \mathrm{~Hz}$ & 11.5166 & $18.8065 \mathrm{~Hz}$ & $17.1442 \mathrm{~Hz}$ & 9.6959 \\
\hline
\end{tabular}

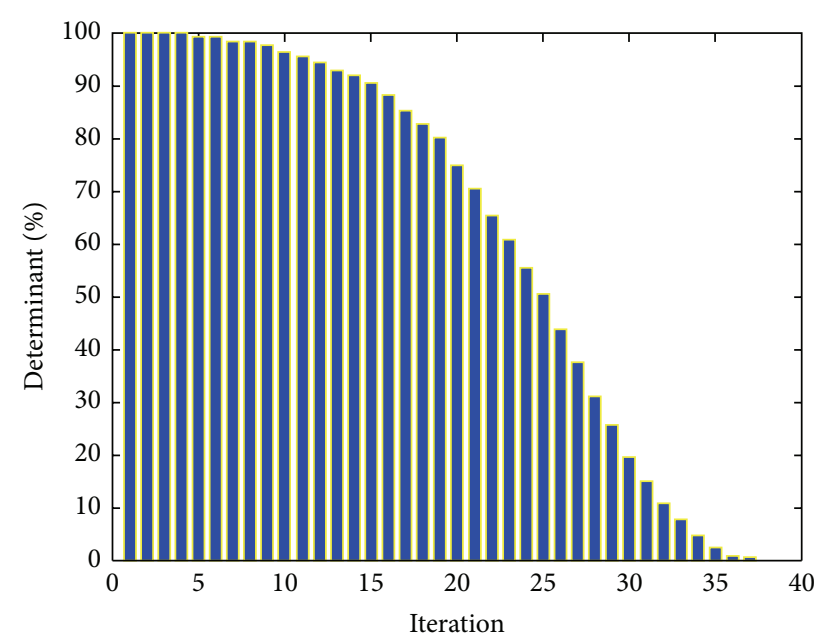

Kinetic energy matrix

FIGURE 7: Kinetic energy matrix values to DOF modification.

Figure 7 illustrates the distribution of kinetic energy for each DOF condition as calculated and derived in this experiment using the KEOT. It can be observed from Figure 7 that as the number of DOF nodes (sensor locations) was gradually reduced, the total kinetic energy contribution of the remaining nodes was also reduced. In the case of the model bridge used in this study, when the number of DOF nodes was reduced to 15 , the kinetic energy contribution was $90 \%$. The contribution of kinetic energy decreased significantly with subsequent removals of DOF nodes. Moreover, when the number of DOF nodes was reduced to the seven optimal sensor locations selected in this study, the kinetic energy contribution was about $10 \%$.

In this study, we conducted SI on a model of a cable-stayed bridge using seven sensors. As described above, the locations of the seven sensors, selected using the KEOT method, were nodes $18,19,21,22,23,25$, and 26 . The total contribution of kinetic energy from these nodes was about $10 \%$. The selection of the seven sensor locations used in this study was verified by the inclusion of the three DOF nodes $(19,22$, and 25) that were considered to be the essential sensor locations for most effectively and simultaneously representing the 1st, 2nd, and 3rd bending modes even at a $92.5 \%$ reduction in the number of DOF nodes. The results derived from the FE model and frequency analysis based on the seven selected sensor locations are shown in Table 7.

Table 7 shows that reducing the number of DOF nodes results in changes in frequency. For quantitative analysis of the changes in frequency, the frequency results and error ratio derived from the initial study with all 39 DOF nodes along with those derived from the modal test are shown in Table 8.

As observed in Table 8, using seven DOF nodes (approximately $82 \%$ reduction of DOF nodes) resulted in an error ratio of approximately $2.7-11.5 \%$ compared to that in the results of FE analysis using all 39 DOF nodes. In addition, using seven DOF nodes resulted in an error ratio of about 3.6-9.6\% compared to the modal test results. The correlation between the mode shape acquired from the FE analysis using seven DOF nodes and the mode shape acquired from the modal test was further evaluated using the modal assurance criterion (MAC) shown in (19) [30]:

$$
\begin{aligned}
& \operatorname{MAC}(A, X) \\
& =\frac{\left|\sum_{j=1}^{n}\left(\Phi_{X}\right)_{j}\left(\Phi_{A}\right)_{j}^{*}\right|^{2}}{\left(\sum_{j=1}^{n}\left(\Phi_{X}\right)_{j}\left(\Phi_{X}\right)_{j}^{*}\right)\left(\sum_{j=1}^{n}\left(\Phi_{A}\right)_{j}\left(\Phi_{A}\right)_{j}^{*}\right)} .
\end{aligned}
$$

Here, $\Phi_{A}$ and $\Phi_{X}$ are the mode shapes as calculated and acquired from the analysis and test, respectively. When the two modes are the same, the MAC value equals 1 , and when the two modes have no correlation, the MAC value equals 0 [31]. Similarly, Ewins [30] points out in his study that the most highly correlated modes will have a MAC value of around 0.9, with MAC values as low as 0.7 considered fair in some cases. $\mathrm{He}$ also asserts that modes with no correlation will have MAC values of around 0.005. Figure 8 and Table 9 illustrate that the mode shapes studied in this research showed satisfactory correlations, but the 3 rd bending mode had a relatively lower correlation than those of the 1st and 2nd modes. 
TABLE 9: MAC values (7 DOF anal. and exp.).

\begin{tabular}{lccc}
\hline & \multicolumn{3}{c}{ FE analysis modes } \\
& 1st & 2nd & 3rd \\
\hline Experimental modes & & & \\
1st & $\mathbf{0 . 9 8 8 6}$ & 0.0000 & 0.4304 \\
2nd & 0.0055 & $\mathbf{0 . 9 9 5 3}$ & 0.0017 \\
3rd & 0.0259 & 0.0000 & $\mathbf{0 . 7 6 6 3}$ \\
\hline
\end{tabular}

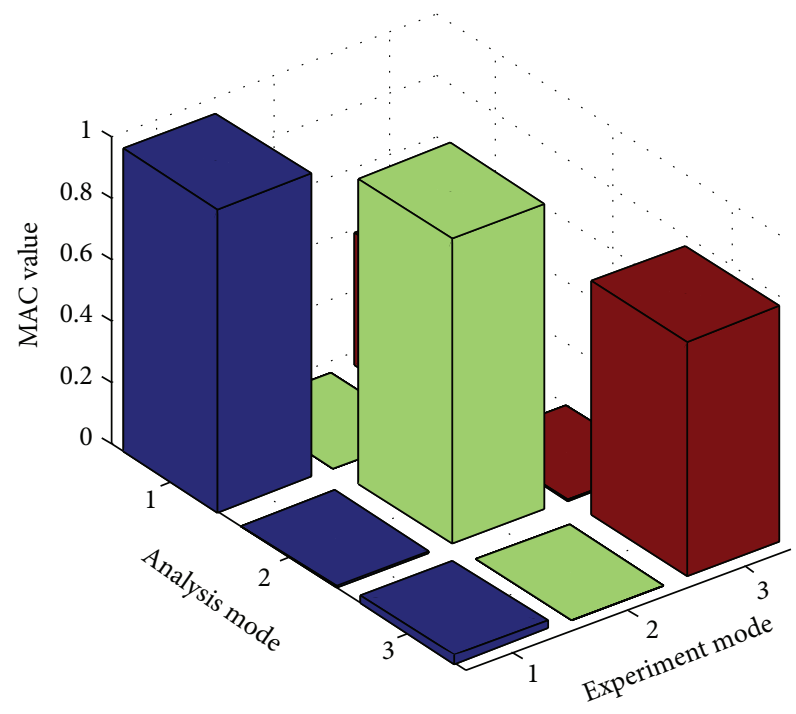

Figure 8: MAC plot (7 DOF anal. and exp.).

The changes in frequency shown in Table 8 and the changes in mode shape shown in Figure 8 and Table 9 are interpretation errors (FE modeling errors) created as a result of reduction of the DOF nodes. To accomplish a thorough SI of a structure as targeted in this study, more accurate modal information needs to be provided regarding the model bridge, and the discrepancies between the analysis results using the seven DOF nodes and the modal test results need to be overcome. Therefore, DMUM was utilized in this study in order to overcome FE modeling errors based on modal test values and to derive a baseline structure that can provide valid modal information.

4.2. FE Model Updating Using DMUM. FE model updating is a technique designed to overcome modeling errors in FE analysis and to secure an accurate model for numerical analysis of the current state of the structure taking into account potential construction errors. In FE model updating, the modal test results derived from the actual structure are applied to the FE analysis results to ultimately create an ideal FE model that contains the modal information of the current structure. This updated FE model can reliably reflect the dynamic behavioral characteristics of the actual structure. The updated model also plays a very important role in acquiring numerical analysis results, which is necessary for accurate assessment of structural states. To achieve FE model updating using DMUM, this study utilized frequency,

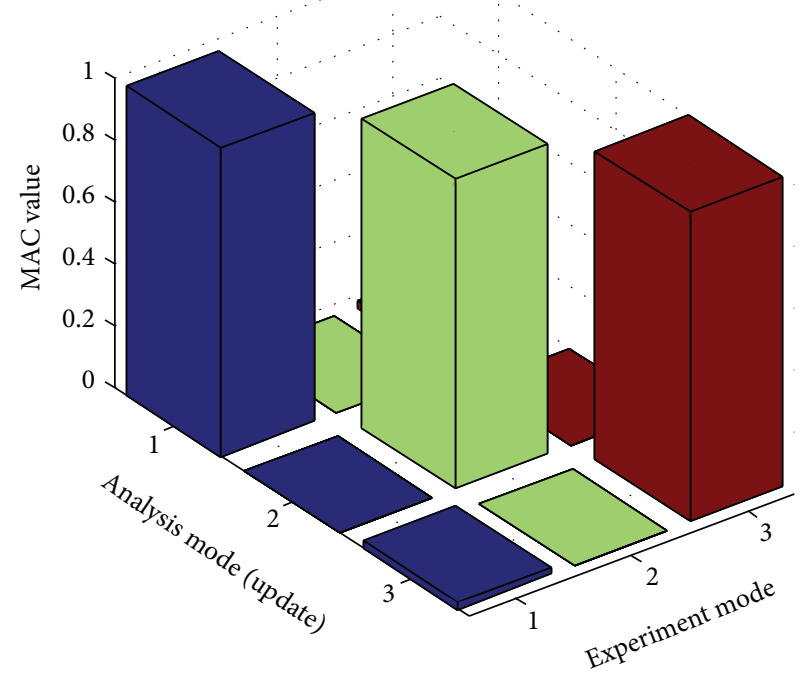

FIGURE 9: MAC plot (update and exp.).

mode shape, and mass and stiffness matrix results derived from the FE analysis along with frequency and mode shape results acquired from the modal test. Table 10 reflects the error ratio between the frequency results derived from the FE model updated using DMUM and the frequency results acquired from the modal test. As shown in Table 10, it was confirmed that the results derived from FE model updating had a significantly lower error rate (close to 0 ).

Next, to verify the effectiveness of DMUM in FE model updating, MAC tests were conducted on the mode shapes. The results of the correlation analysis between the mode shapes derived from the updated FE model and those derived from the modal test are as shown in Figure 9 and Table 11. The results show that using DMUM to update the FE model results in a MAC value of " 1 ," thus demonstrating an outstanding correlation with the modal test results.

In addition, in order to visualize the mode correlation evaluated using MAC and to evaluate the correlation between mode shapes before and after FE model updating, the mode shapes derived from analysis using seven DOF nodes or sensor locations and the mode shapes derived from the updated FE model were graphed as shown in Figure 10. As the graphs illustrate, the mode shape from the updated FE model follows the mode shape of the modal test, and not the mode shape of the FE analysis. This verifies the fact that by incorporating the modal information of the structure, FE model updating not only provides more accurate frequency values but also provides more accurate mode shape patterns. Ultimately, this proves that FE model updating using DMUM can overcome modeling errors and it is an effective way to create an accurate model for numerical analysis that can take into account the actual state of the structure.

\section{Conclusion}

The aim of this study was to research the formulation of a baseline structure through optimal sensor location selection 
TABLE 10: Comparison of experimental results and updating results.

\begin{tabular}{lccc}
\hline & \multicolumn{2}{c}{ Analysis and experimental results using EOT } & Error ratio \\
& FE model update & Experimental & 0.0110 \\
1st mode & $9.0880 \mathrm{~Hz}$ & $9.0890 \mathrm{~Hz}$ & 0.0124 \\
2nd mode & $11.2543 \mathrm{~Hz}$ & $11.2557 \mathrm{~Hz}$ & 0.0139 \\
3rd mode & $17.1418 \mathrm{~Hz}$ & $17.1442 \mathrm{~Hz}$ & \\
\hline
\end{tabular}

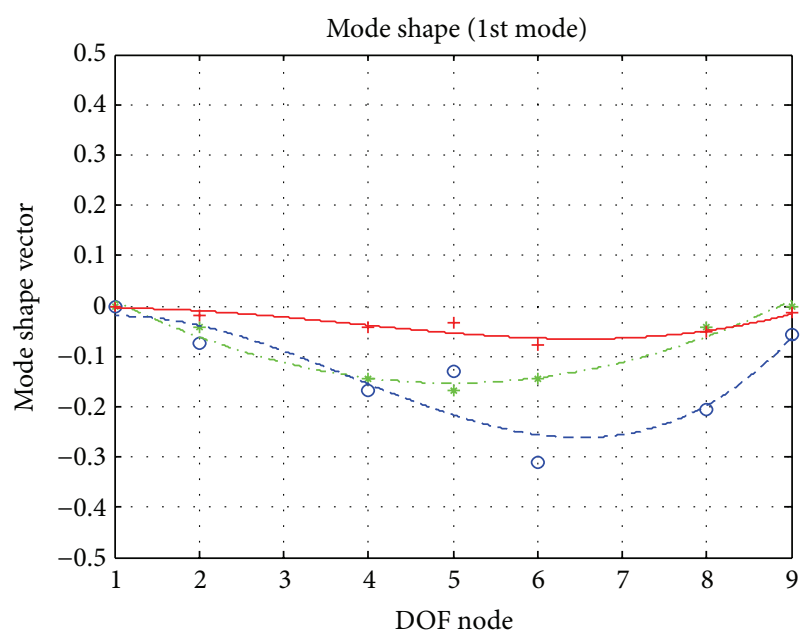

\footnotetext{
* Analysis mode shape vector -.-- Analysis mode shape curve

+ Experiment mode shape vector — Experiment mode shape curve

- Update mode shape vector -- - Update mode shape curve
}

(a) 1st bending mode shape

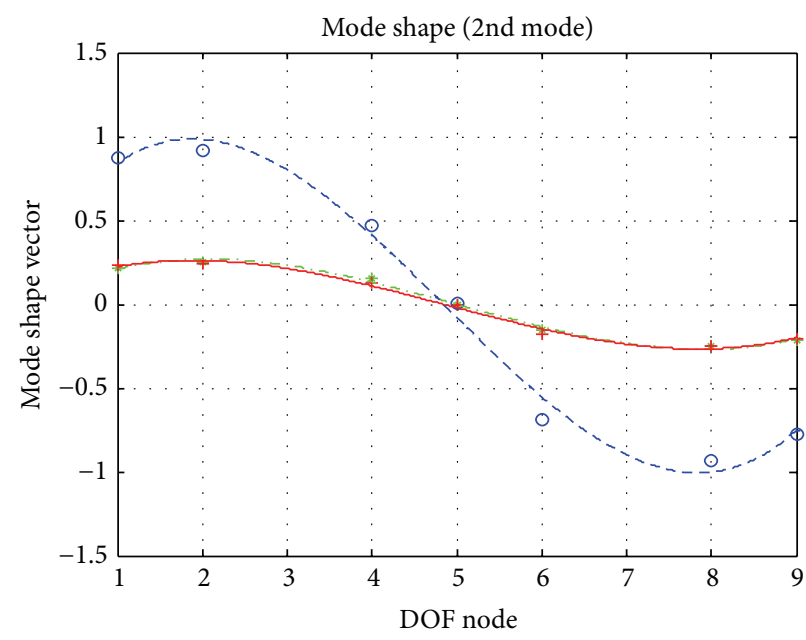

$\begin{array}{ll}\text { * Analysis mode shape vector } & - \text {-. Analysis mode shape curve } \\ \text { + Experiment mode shape vector } & \text { - Experiment mode shape curve } \\ \text { - Update mode shape vector } & \text {-- - Update mode shape curve }\end{array}$

(b) 2nd bending mode shape

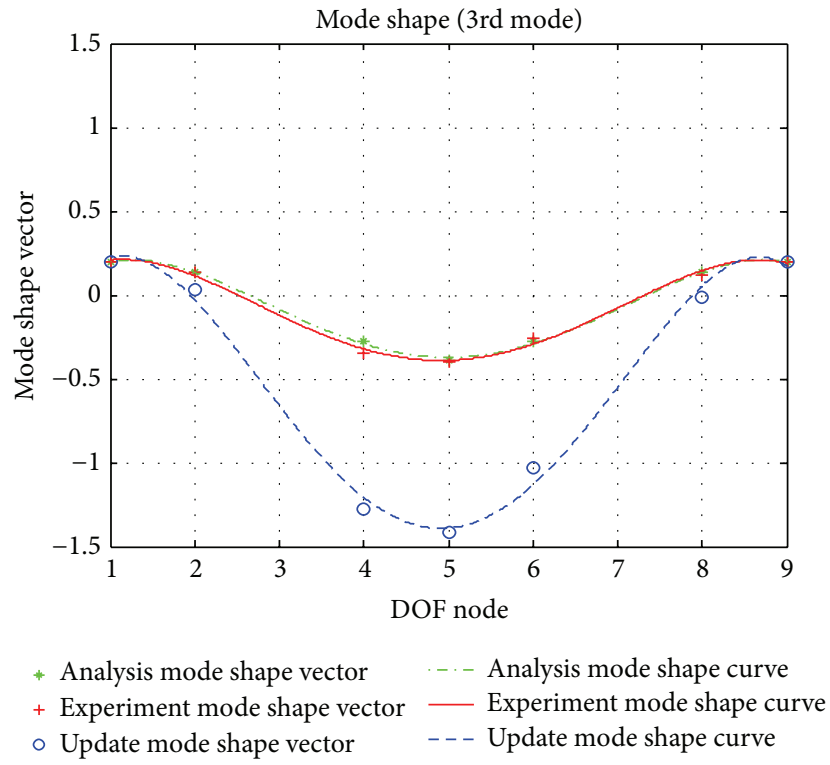

(c) 3rd bending mode shape

FIGURE 10: Comparison of 7 DOF analysis, updating, and experimental mode shapes.

and FE model updating for conducting SI on cable-stayed bridges. The conclusions are as follows:

(1) By comparing the contribution of each DOF node to the total kinetic energy and progressively removing those that do not contribute based on the target modes, the KEOT method was proven to be an effective approach for selecting the optimal number and location of sensors among the multiple sensor locations (DOF) in order to acquire the desired 
TABLE 11: MAC values (updating and exp.).

\begin{tabular}{lccc}
\hline & \multicolumn{3}{c}{ FE analysis modes (update) } \\
& 1st & 2nd & 3rd \\
\hline $\begin{array}{l}\text { Experiment } \\
\text { modes } \\
\text { 1st }\end{array}$ & & & \\
2nd & $\mathbf{1 . 0 0 0 0}$ & 0.0003 & 0.0225 \\
3rd & 0.0002 & $\mathbf{1 . 0 0 0 0}$ & 0.0005 \\
\hline
\end{tabular}

responses. In this study, using seven sensors out of a total of 39 sensor locations to consider the three lowest bending modes (about 20\% of the total) was sufficient to effectively acquire relevant structural information on the cable-stayed bridge.

(2) In addition, by applying structural information measured from the actual structure and constructing a corresponding FE model to overcome initial errors in FE modeling, the DMUM method was proven to be an effective method for defining a baseline structure that incorporates the behavioral characteristics of the actual structure. DMUM has particularly strong potential for practical use from temporal and economical standpoints because it calculates stiffness and mass values from just a single matrix operation as opposed to other methods that require repetitive operations such as EMUM.

(3) In conclusion, for structures such as cable-stayed bridges where the characteristic flexing behavior creates low and short-interval frequencies, situations where multiple sensor locations (DOF) are required because of structural complexity, and situations where SHM and evaluation and maintenance needs to be performed on structures with low and short-interval frequencies, the use of both KEOT and DMUM can be the most effective approach for conducting SI from the standpoints of both economy and usability because they provide accurate results with minimum number of measurement locations.

\section{Conflict of Interests}

The authors declare that there is no conflict of interests regarding the publication of this paper.

\section{Acknowledgments}

This research was supported by the Basic Science Research Program through the National Research Foundation of Korea (NRF) funded by the Ministry of Education, Science and Technology (Grant no. NRF-2013R1A2A1A01016192) and funded by the Ministry of Science, ICT and Future Planning (Grant no. NRF-2013R1A1A1063540).

\section{References}

[1] S. C. Lie and J. T. P. Yao, "Structural identification concept," Journal of the Structural Division, vol. 104, no. 12, pp. 1845-1858, 1978.

[2] G. F. Sirca Jr. and H. Adeli, "System identification in structural engineering," Scientia Iranica A, vol. 19, no. 6, pp. 1355-1364, 2012.

[3] V. Arora, "Structural damping identification method using normal FRFs," International Journal of Solids and Structures, vol. 51, no. 1, pp. 133-143, 2014.

[4] Z. D. Zheng, Z. R. Lu, W. H. Chen, and J. K. Liu, "Structural damage identification based on power spectral density sensitivity analysis of dynamic responses," Computers \& Structures, vol. 146, pp. 176-184, 2015.

[5] D. Dessi and G. Camerlengo, "Damage identification techniques via modal curvature analysis: overview and comparison," Mechanical Systems and Signal Processing, vol. 52-53, pp. 181205, 2015.

[6] C. Farrar and P. J. Cornwell, "Structural health monitoring studies of the alamosa canyon and I-40 bridges," Tech. Rep. LA13635-MS, Los Alamos National Laboratory, Los Alamos, NM, USA, 1998.

[7] F. Benedettini, M. Dilena, and A. Morassi, "Vibration analysis and structural identification of a curved multi-span viaduct," Mechanical Systems and Signal Processing, vol. 54-55, pp. 84-107, 2015.

[8] H. Chen, M. Kurt, Y. S. Lee, D. M. McFarland, L. A. Bergman, and A. F. Vakakis, "Experimental system identification of the dynamics of a vibro-impact beam with a view towards structural health monitoring and damage detection," Mechanical Systems and Signal Processing, vol. 46, no. 1, pp. 91-113, 2014.

[9] M. L. Wang, G. Heo, and D. Satpathi, "Dynamic characterization of a long span bridge: a finite element based approach," Soil Dynamics and Earthquake Engineering, vol. 16, no. 7-8, pp. 503512, 1997.

[10] M. L. Wang, G. Heo, and D. Satpathi, "A health monitoring system for large structural systems," Smart Materials and Structures, vol. 7, no. 5, pp. 606-616, 1998.

[11] G. Heo, M. L. Wang, and D. Satpathi, "Optimal transducer placement for health monitoring of long span bridge," Soil Dynamics and Earthquake Engineering, vol. 16, no. 7-8, pp. 495$502,1997$.

[12] H. Gwanghee, An automated health monitoring system for large civil structural system [Ph.D. thesis], University of New Mexico, Albuquerque, NM, USA, 1997.

[13] D. C. Kammer, "Effect of model error on sensor placement for on-orbit modal identification of large space structures," Journal of Guidance, Control, and Dynamics, vol. 15, no. 2, pp. 334-341, 1992.

[14] F. E. Udwadia, "Methodology for optimum sensor locations for parameter identification in dynamic systems," Journal of Engineering Mechanics, vol. 120, no. 2, pp. 368-387, 1994.

[15] A. R. M. Rao, K. Lakshmi, and S. Krishnakumar, "A generalized optimal sensor placement technique for structural health monitoring and system identification," Procedia Engineering, vol. 86, pp. 529-538, 2014.

[16] Z. Y. Shi, S. S. Law, and L. M. Zhang, "Optimum sensor placement of structural damage detection," Journal of Engineering Mechanics, vol. 126, no. 11, pp. 1173-1179, 2000. 
[17] T. Pothisiri and K. D. Hjelmstad, "Structural damage detection and assessment from modal response," Journal of Engineering Mechanics, vol. 129, no. 2, pp. 135-145, 2003.

[18] M. Ge and E. M. Lui, "Structural damage identification using system dynamic properties," Computers and Structures, vol. 83, no. 27, pp. 2185-2196, 2005.

[19] T. P. Waters, Finite element model updating using measured frequency response functions [Ph.D. thesis], Department of Aerospace Engineering, University of Bristol, Bristol, UK, 1995.

[20] Q. W. Zhang, C. C. Chang, and T. Y. P. Chang, "Finite element model updating for structures with parametric constraints," Earthquake Engineering and Structural Dynamics, vol. 29, no. 7, pp. 927-944, 2000.

[21] J. E. Mottershead and M. I. Friswell, "Model updating in structural dynamics: a survey," Journal of Sound and Vibration, vol. 167, no. 2, pp. 347-375, 1993.

[22] M. I. Friswell and J. E. Mottershead, Finite Element Model Updating in Structural Dynamics, vol. 38 of Solid Mechanics and its Applications, Kluwer Academic Publishers, Dordrecht, The Netherlands, 1995.

[23] M. Baruch, "Optimization procedure to correct stiffness and flexibility matrices using vibration tests," AIAA Journal, vol. 16, no. 11, pp. 1208-1210, 1978.

[24] A. Berman and E. J. Nagy, "Improvement of a large analytical model using test data," AIAA Journal, vol. 21, no. 8, pp. 1168$1173,1983$.

[25] J. Sidhu and D. J. Ewins, "Correlation of finite element and modal test studies of a practical structure," in Proceedings of the 2nd International Modal Analysis Conference, pp. 156-162, Orlando, Fla, USA, February 1984.

[26] D. J. Ewins, J. He, and N. A. J. Lieven, "A review of the error matrix method (EMM) for structural dynamic model comparison," in Proceedings of the ESA Symposium on the Applications of Advanced Structural Materials, Noordwijk, The Netherlands, 1988.

[27] J.-S. Fuh and A. Berman, "Comment on 'Stiffness matrix adjustment using mode data,' AIAA Journal, vol. 24, no. 8, pp. 1405-1406, 1986.

[28] A. Berman, "Mass matrix correction using an incomplete set of measured modes," AIAA Journal, vol. 17, no. 10, pp. 1147-1148, 1979.

[29] R. J. Guyan, "Reduction of stiffness and mass matrices," AIAA Journal, vol. 3, no. 2, p. 380, 1965.

[30] D. J. Ewins, Modal Testing: Theory, Practice and Application, Research Studies Pre, 2000.

[31] G. Heo, J. Jeon, C. O. Lee, G. Lee, and W. Lee, "FE model updating for health monitoring of structures and its experimental verification by damage detection," Key Engineering Materials, vol. 321-323, pp. 268-272, 2006. 


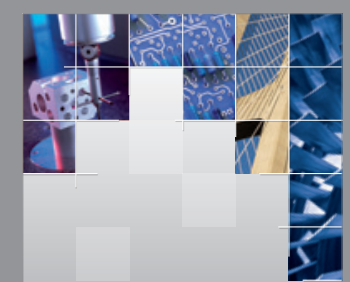

\section{Enfincering}
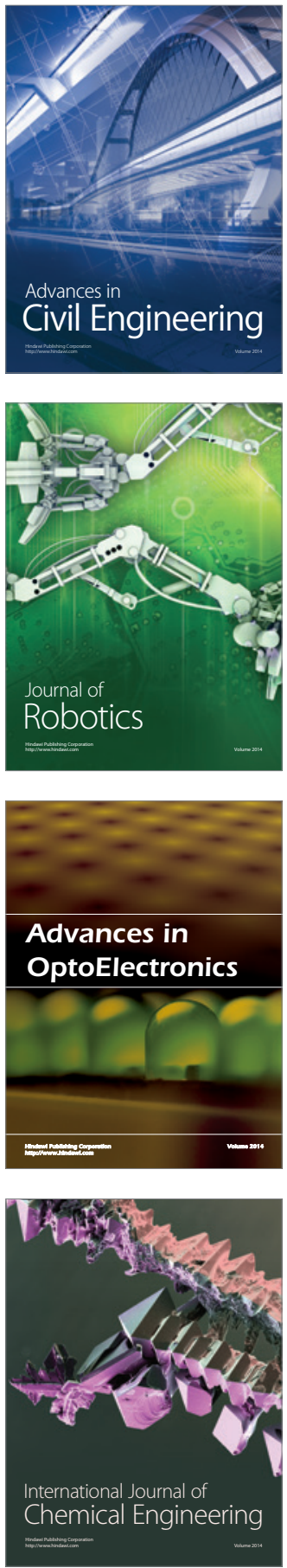

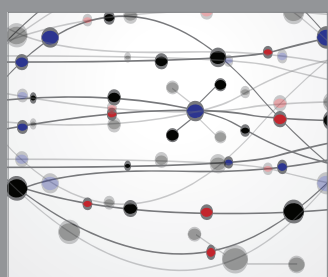

The Scientific World Journal

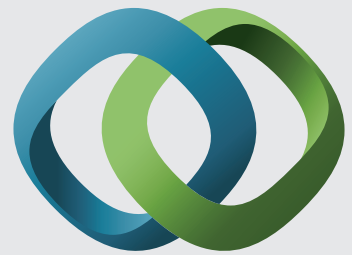

\section{Hindawi}

Submit your manuscripts at

http://www.hindawi.com
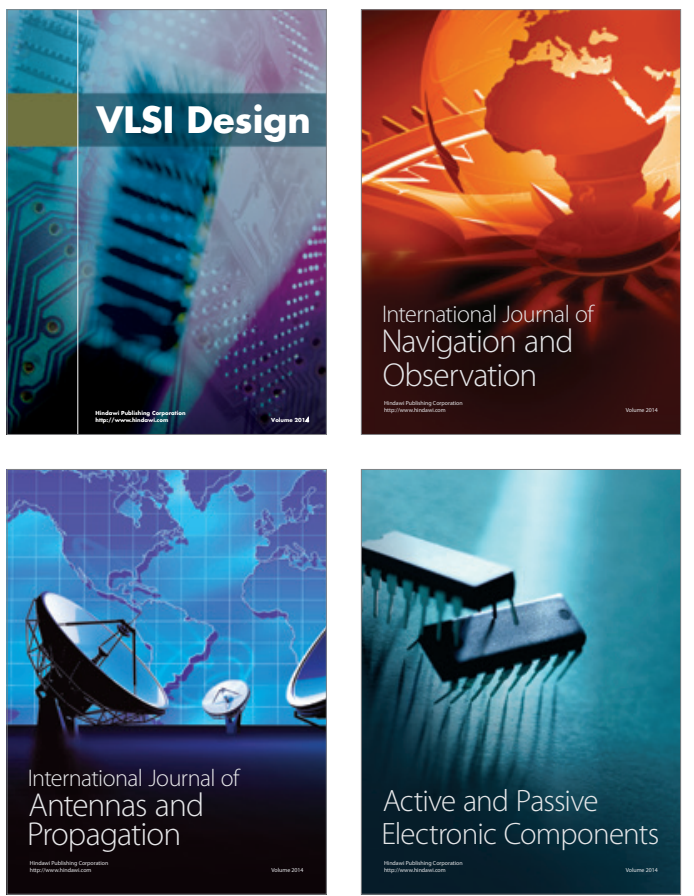
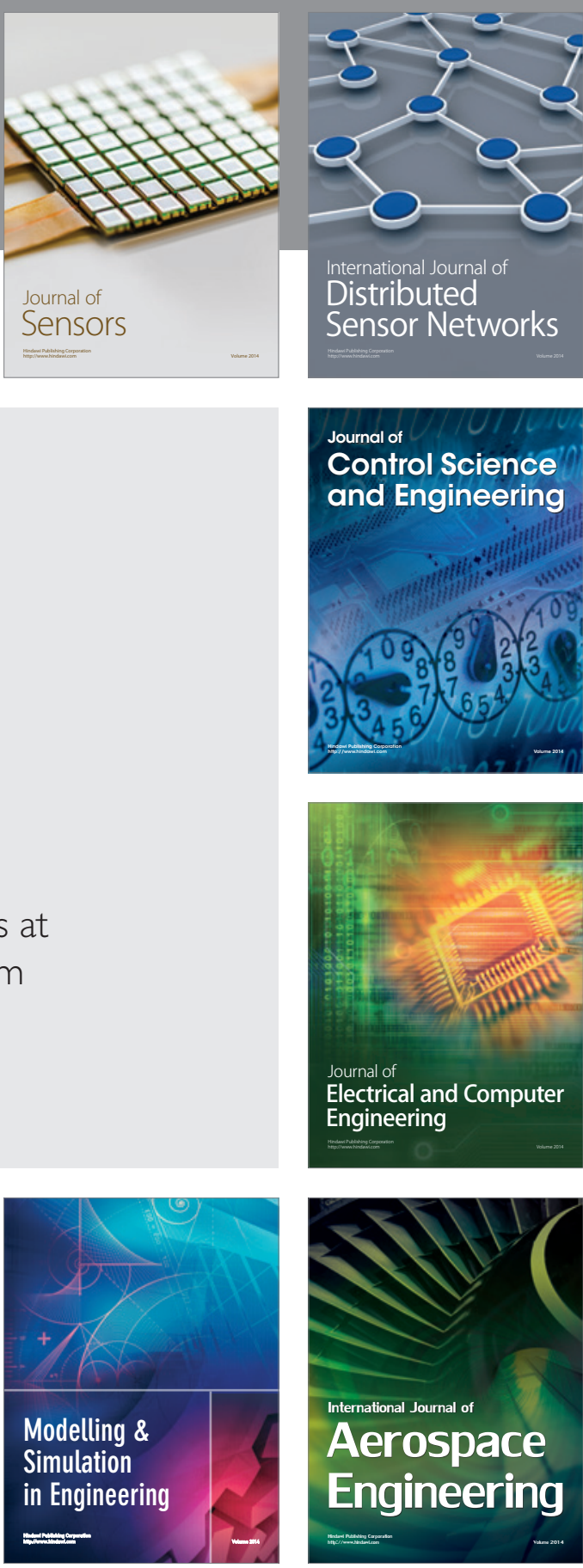

International Journal of

Distributed

Sensor Networks

Journal of

Control Science

and Engineering
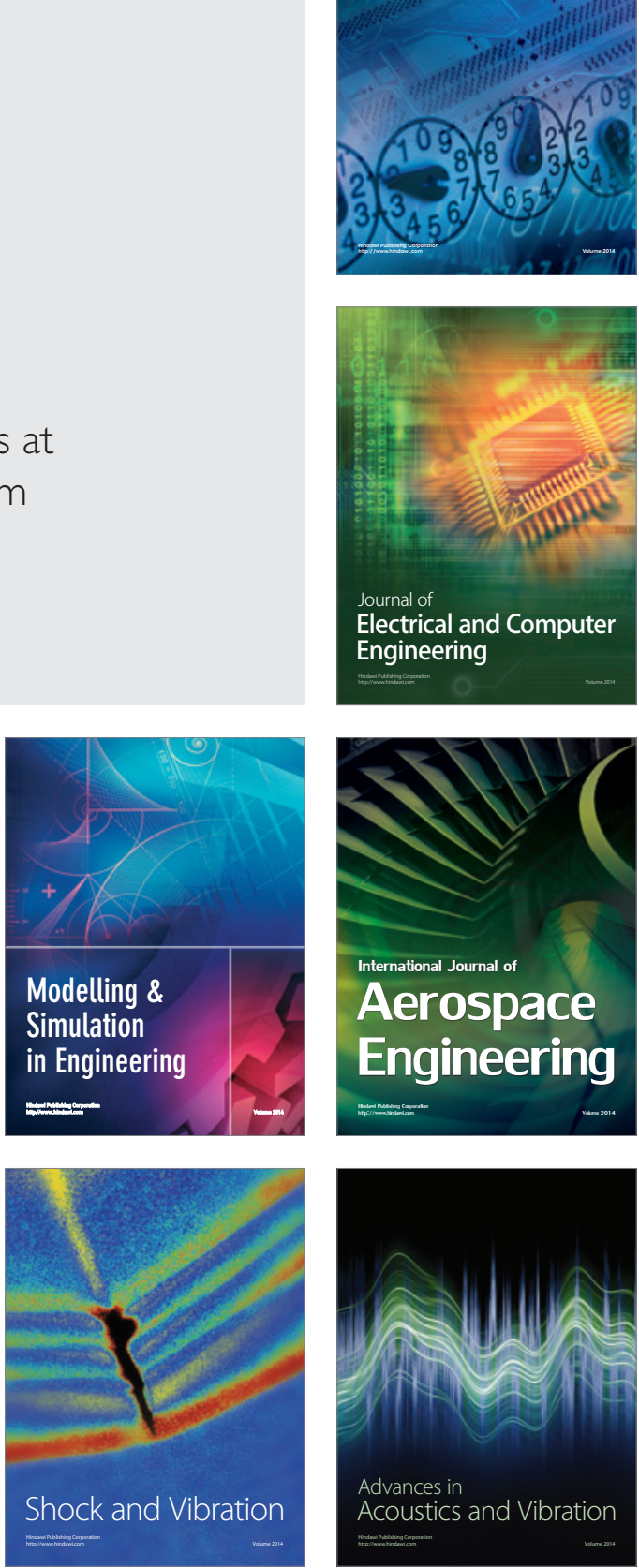